\title{
PROBING COOL AND WARM INFRARED GALAXIES USING PHOTOMETRIC AND STRUCTURAL MEASURES
}

\author{
Nurur Rahman, ${ }^{1}$ George Helou, and Joseph M. Mazzarella \\ Infrared Processing and Analysis Center, California Institute of Technology, Mail Code 100-22, 770 South Wilson Avenue, Pasadena, \\ CA 91125; nurur@ipac.caltech.edu,gxh@ipac.caltech.edu,mazz@ipac.caltech.edu \\ Received 2006 July 10; accepted 2006 August 16
}

\begin{abstract}
We have analyzed a sample of nearby cool and warm infrared (IR) galaxies using photometric and structural parameters. The set of measures include far-infrared color $\left[C=\log \left(S_{60 \mu \mathrm{m}} / S_{100 \mu \mathrm{m}}\right)\right]$, total IR luminosity $\left(L_{\mathrm{TIR}}\right)$, radio surface brightness, and radio, near-infrared, and optical sizes. In a given luminosity range cool and warm galaxies are considered as those sources that are found approximately $1 \sigma$ below and above the mean color in the farinfrared $C-L_{\mathrm{TIR}}$ diagram. We find that galaxy radio surface brightness is well correlated with color whereas size is less well correlated with color. Our analysis indicates that IR galaxies that are dominated by cool dust are large, massive spirals that are not strongly interacting or merging and presumably the ones with the least active star formation. Dust in these cool objects is less centrally concentrated than in the more typical luminous and ultraluminous IR galaxies that are dominated by warm dust. Our study also shows that low-luminosity early-type unbarred and transitional spirals are responsible for the large scatter in the $C-L_{\mathrm{TIR}}$ diagram. Among highly luminous galaxies, late-type unbarred spirals are predominately warm, and early-type unbarred and barred spirals are systematically cooler. We highlight the significance of the $C-L_{\mathrm{TIR}}$ diagram in terms of local and high-redshift submillimeter galaxies.
\end{abstract}

Subject headings: galaxies: active — galaxies: general — galaxies: spiral — galaxies: starburst — galaxies: structure

Online material: machine-readable table

\section{INTRODUCTION}

Observations by the Infrared Astronomical Satellite (IRAS) led to the identification of very luminous and ultraluminous infrared galaxies, commonly known as LIRGs and ULIRGs, respectively. These galaxies have enormous far-infrared (FIR) luminosities, $10^{11}<L_{\mathrm{FIR}} / L_{\odot}<10^{12}$ for LIRGs and $L_{\mathrm{FIR}}>10^{12} L_{\odot}$ for ULIRGs, and emit the bulk of their energy at the infrared wavelengths (Soifer et al. 1984, 1986, 1987; Sanders et al. 1988). Subsequent multiwavelength studies reveal that in the local universe $(z<0.1)$ ULIRGs have total luminosities ( $\left.L_{\mathrm{TIR}}\right)$ comparable to and space densities higher than those of optical quasars (Sanders et al. 1988; Kim \& Sanders 1998).

Understanding the nature and origin of energy sources in LIRGs and ULIRGs has been the subject of much debate. Studies indicate that in the vast majority of these objects power comes from dust that is heated to various temperatures by various thermal and nonthermal processes such as ongoing (steady state) star formation, intense starburst phases, and/or synchrotron radiation from supernovae explosions (Lawrence et al. 1986; Genzel et al. 1998; Lutz et al. 1998). These systems may also be powered by active galactic nuclei (AGNs; Lonsdale et al. 1993). The energy distributions suggest that most of the observed FIR emission from luminous disk galaxies is due to the thermal radiation from warm dust heated by hot stars embedded in $\mathrm{H}$ in regions and molecular clouds heated directly by young OB stars, and cool dust from the general interstellar radiation field (Helou 1986; Lonsdale Persson \& Helou 1987; Barvainis et al. 1992). Dust could also be heated by shocks in the interstellar medium during the collision or interaction of galaxies (Harwit et al. 1987).

Dust heating mechanisms can be traced indirectly as each of these should correspond to particular spatial distributions of the

\footnotetext{
${ }^{1}$ National Research Council Postdoctoral Fellow.
}

FIR light. For example, the FIR emission originating in galaxies dominated by an AGN would appear compact and unresolved. On the other hand, starburst-heated dust should have about the same scale size as the burst itself because young stars are well mixed with the gas from which stars are forming. Dust heated by the nonionizing photons from cold, Population I stars might be expected to follow the smoothed distribution of older giants in the galaxy (Zink et al. 2000). A determination of the total energy output such as luminosity, the amount of dust present in the interstellar medium, and its spatial distribution within the galaxies, as well as its relationship to other basic components, such as the atomic and molecular gas and the stars, is essential to studies connecting galactic structure and nature of the IR energy sources (Carico et al. 1990; Sopp \& Alexander 1991, 1992; Andreani \& Franceschini 1992, 1996; Alton et al. 1998; Domingue et al. 1999; Siebenmorgen et al. 1999; Haas et al. 2000; Stickel et al. 2000; Trewhella et al. 2000; Zink et al. 2000).

The FIR color $C$, defined by the IRAS $60 \mu \mathrm{m}$-to-100 $\mu \mathrm{m}$ flux density ratio, an indicator of characteristic dust temperature, is a diagnostic of the typical heating conditions in the interstellar medium of a galaxy (Bothun et al. 1989; Soifer \& Neugebauer 1991). It has been shown that low-redshift IRAS galaxies exhibit positive correlation of $C$ with $L_{\mathrm{FIR}}$ such that more luminous galaxies tend to be warmer as compared to less luminous galaxies (Soifer et al. 1987; Dale et al. 2001; see Fig. 1 in this study). The color changes systematically over roughly 3 orders of magnitude in luminosity with large scatter. As a result, there is a substantial number of highly luminous but cool galaxies as well as lowluminosity yet warm/hot galaxies (Dale et al. 2001; Chapman et al. 2003). In this study we attempt to understand cool and warm IR-luminous sources in terms of the geometric distribution of dust and galaxy morphology. In a given FIR luminosity range, these galaxies are considered in the context wherein cool galaxies are $1 \sigma$ below and warm galaxies are $1 \sigma$ above the 


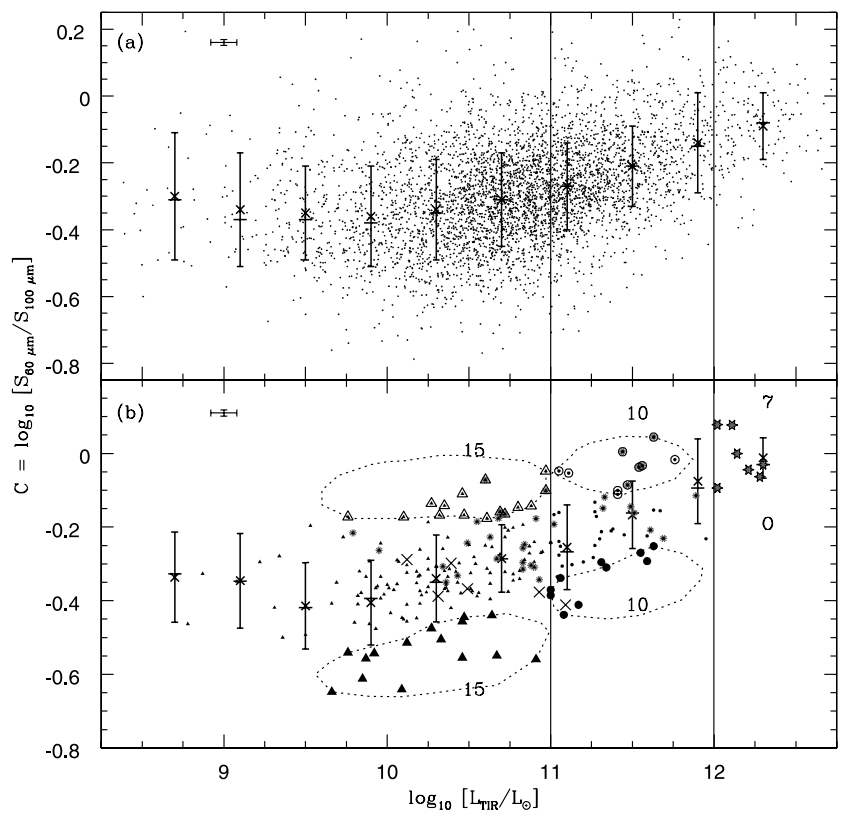

FIG. 1.-FIR color as a function of $L_{\mathrm{TIR}}$ for $(a)$ the $I R A S S_{60 \mu \mathrm{m}}>1.2 \mathrm{Jy}$ sample of $\sim 4350$ galaxies and $(b)$ our sample of 213 galaxies. Three classes of galaxies, in general, are shown by small triangles (SFGs), small dots (LIRGs), and stars (ULIRGs). The cool and warm sources are indicated, respectively, by large filled symbols and open symbols (triangles for SFGs and circles for LIRGs) in the regions delineated by dotted closed curves. In each luminosity bin the horizontal bar and (small) cross represent median and mean color. Each luminosity class (delineated by long vertical lines) contains equal number of cool and warm sources: $15+15$ for SFGs and 10+10 for LIRGs. Large crosses and asterisks represent, respectively, local submillimeter and interacting/disturbed galaxies. A representative error bar is shown at the top of each panel.

mean color at a given luminosity. We are interested in differences between cool and warm galaxies in the same luminosity range that might suggest differences in the nature of the heating sources.

In an interesting study Young (1999) showed that star formation efficiency (SFE), defined by the ratio of $L_{\mathrm{TIR}}$ to molecular hydrogen mass $M_{\mathrm{H}_{2}}$, is anticorrelated with disk (optical) sizes. The trend is prevalent in galaxies of various Hubble types and environments. It has been attributed to the shear present in disk galaxies. The molecular clouds in larger disks would experience increased turbulence, which would reduce the efficiency of star formation (Young 1999). Galaxies in Young's sample span a broad range in FIR color comprising normal star-forming galaxies (SFGs; $L_{\mathrm{FIR}} / L_{\odot}<10^{11}$ ), as well as LIRGs and ULIRGs. In Young's study, however, it remains unexplored how cool and warm systems behave in the visual as well as in the longer wavelengths. Since the IR-luminous galaxies contain very large amounts of dust, the interpretation of any observation at optical or shorter infrared wavelengths gets complicated by extinction. Therefore, to probe the origin of large IR luminosity, it is necessary to have knowledge of the spatial extents of the emitting regions in observations unaffected by dust extinction.

The paper is organized as follows. We describe the data in $\S 2$ and parameter error estimation in $\S 3$. We present our results in $\S 4$ and discuss the implications of our results in $\S 5$. The conclusions are given in $\S 6$.

\section{DATA}

$I R A S$ resolved only the largest and nearest galaxies because of its comparatively large beam size of $\sim 2^{\prime}-4^{\prime}$ at 60 and $100 \mu \mathrm{m}$. Therefore, the FIR brightness distributions of most of the sources detected by $I R A S$ are unknown. In contrast to the rather large scatter in optical-FIR correlations, a tight correlation of FIR with radio continuum total flux densities for infrared-selected galaxies appears to hold locally within individual galaxies (Helou et al. 1985; Beck \& Golla 1988; Bicay et al. 1989; Murphy et al. 2006). In nearby galaxies where (IRAS) FIR sizes have been measured directly, radio sizes are known to match, or to be somewhat larger than the size scale in the FIR (Bicay \& Helou 1990). Besides, FIR and radio continuum brightness distributions of these galaxies show remarkable similarity (Marsh \& Helou 1995). The agreement in FIR and radio sizes, albeit derived from a smaller sample, suggests that high-resolution radio maps may be taken as a good substitute, or at least as an upper limit for the size of the unobtainable FIR maps. We therefore use $1.49 \mathrm{GHz}$ radio size as a proxy for the size of FIR emission.

Wang \& Helou (1992, hereafter WH92) studied the compactness of FIR-bright galaxies and constructed a list of 330 galaxies from the IRAS Bright Galaxy Sample (BGS; Soifer et al. 1986, 1987, 1989). WH92 constructed a flux-limited sample of extragalactic objects brighter than $5.24 \mathrm{Jy}$ at $60 \mu \mathrm{m}$, covering the entire sky surveyed by $\operatorname{IR} A S$ at Galactic latitude $|b|>30^{\circ}$. For radio data (rest-frame flux density, size), WH92 used the atlas of the $1.49 \mathrm{GHz}$ radio maps based on VLA observations of IR-bright galaxies that had been compiled by Condon et al. (1991, hereafter CAH91). After removing AGN-dominated galaxies identified as highly compact radio sources, the final list of WH92 contained 218 galaxies. Our sample is based on this list of 218 galaxies. We use the same radio data; however, we take the restframe IRAS flux densities, luminosity, and estimated distance of member galaxies from the Revised Bright Galaxy Sample (RBGS) since it provides the best available reference for accurate IRAS fluxes and IR luminosities of galaxies in the local universe (see Sanders et al. 2003 for details on the RBGS).

Particular attention is given to exclude AGNs from the sample. Although the list of WH92 was carefully compiled to get rid of the radio monsters, we have checked the entire sample following the prescription of de Grijp et al. (1985) using mid- and far-infrared color-color correlations. We have found five galaxies that fall into the region of the color-color plot that is mostly occupied by AGNlike sources (de Grijp et al. 1985). We have removed them from the list. The radio fluxes of the remaining 213 galaxies correlate well with the IRAS fluxes, and all galaxies fall within $\sim 1.5 \sigma$ of the mean $q$-value in the $L_{\mathrm{FIR}}-q$ correlation, indicating absence of radio excess objects (the $q$-parameter is defined in CAH91). We have also made visual inspection of the observed spectral energy distributions of member galaxies. From this analysis we conclude that our final sample of 213 sources are star-forming disk galaxies, although there may be AGNs present that are not energetically dominant in the far-infrared.

The number of SFGs, LIRGs, and ULIRGs in our sample is 154,52 , and 7 , respectively. These three classes of galaxies have the following redshift distributions: $0.0<z<0.016,0.012<$ $z<0.051$, and $0.018<z<0.082$ with the median redshifts $0.006,0.023$, and 0.055 . The effective color temperature of the integrated dust emission in the sample galaxies ranges between $\sim 25$ and $40 \mathrm{~K}$, assuming emissivity index $\beta=2$ (Dunne et al. 2000; Dunne \& Eales 2001). The ULIRGs show signs of interaction, e.g., the presence of neighbors, and accretion, e.g., tidal tails and disturbed outer envelopes. The majority of LIRGs appear as single, isolated systems, while $30 \%$ of them appear disturbed. At the lower end of the luminosity range, the LIRGs are luminous single isolated galaxies, with features resembling low-luminosity SFGs. A minority of SFGs (15\%) have disturbed outer envelopes or nearby companions. It should be noted that our sample is in 
TABLE 1

Partial List of Low-Luminosity Star-forming Galaxies

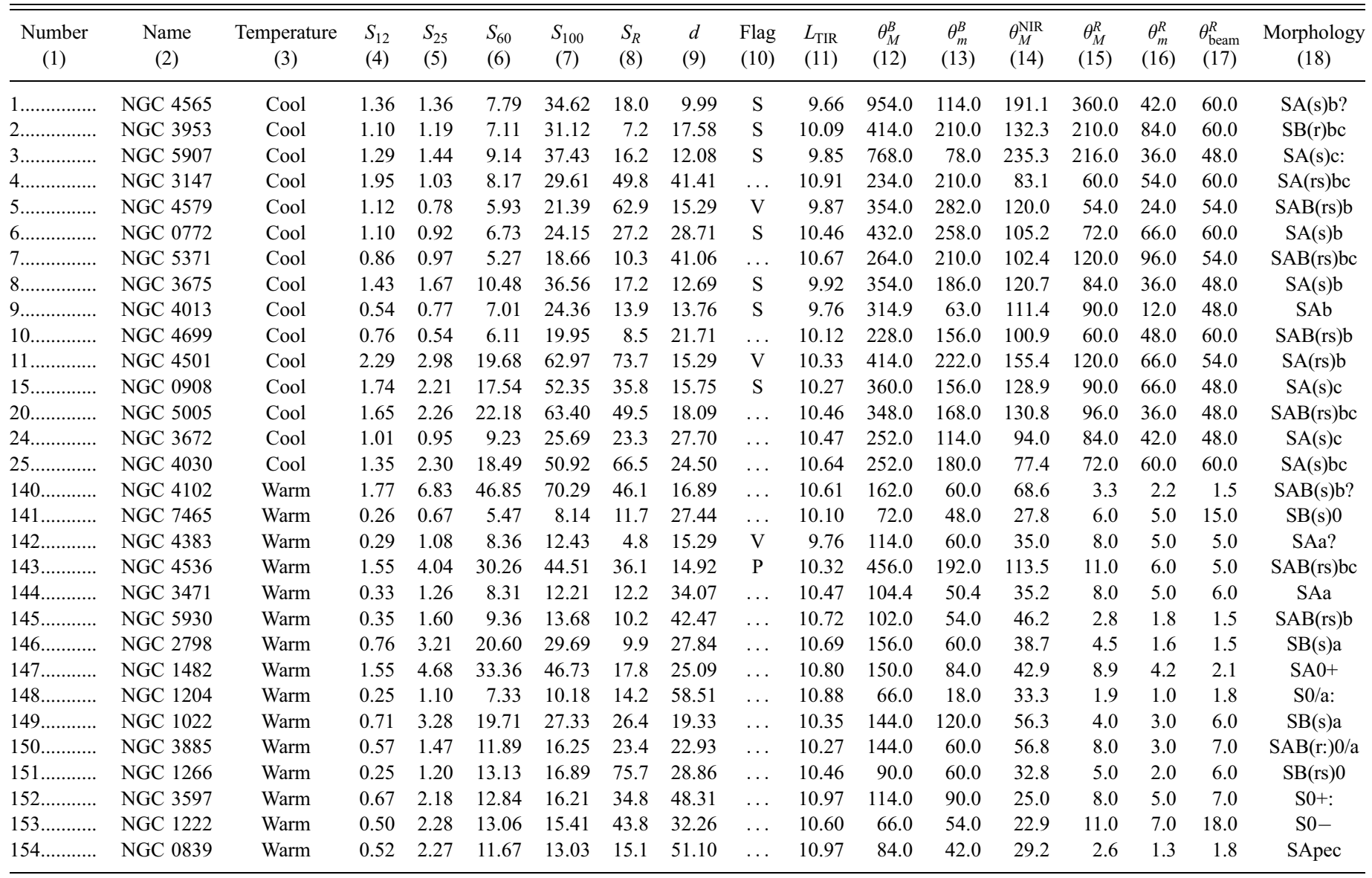

Notes.-Col. (1): Number. Col. (2): Galaxy name. Col. (3): Dust temperature. Cols. (4)-(7): IRAS flux densities (Jy) from RBGS (Sanders et al. 2003). Col. (8): 1.49 GHz flux density (mJy) (peak flux $S_{P}$ in CAH91 and WH92). Col. (9): Estimated galaxy distance $d$ in Mpc. Col. (10): Flags P, S, and Von distance estimate (see RBGS Table 1 for details). Col. (11): Total IR luminosity $\log \left(L_{\mathrm{TIR}}\right)$ in $L_{\odot}$ (RBGS). Cols. (12)-(13): $B$-band $25 \mathrm{mag} \mathrm{arcsec}^{-2}$ major and minor axes (arcsec). Col. (14): $K_{s}$-band $20 \mathrm{mag}$ arcsec ${ }^{-2}$ major axis (arcsec). Cols. (15)-(16): $1.49 \mathrm{GHz}$ major and minor axes for the resolution at which the galaxy is resolved (arcsec). Col. (17): 1.49 GHz beam resolution. Col. (18): Galaxy optical morphology. Table 1 is published in its entirety in the electronic edition of the Astrophysical Journal. A portion is shown here for guidance regarding its form and content.

no sense complete or free of selection bias. The objective is to have a sample large enough to carry out a statistical study on photometric and structural properties of cool and warm galaxies.

The NASA/IPAC Extragalactic Database (NED) and the LyonMeudon Extragalactic Database (LEDA) have been used to obtain Galactic-extinction-corrected $B$-band luminosity $L_{B}$ and $25 \mathrm{mag}$ $\operatorname{arcsec}^{-2}$ linear diameter $D_{B}$. The near-infrared (NIR) $K_{S}$-band $(2.17 \mu \mathrm{m})$ magnitude of our sample is $5.0<K_{s}<10.6$. For bright galaxies Jarrett et al. (2000) have recommended $K_{s}$-band $20 \mathrm{mag} \operatorname{arcsec}^{-2}$ diameter $D_{\mathrm{NIR}}$ as the most reliable and robust for galaxy photometry. We obtained $D_{\mathrm{NIR}}$ from Two Micron All Sky Survey catalog (Jarrett 2000) using a $5^{\prime \prime}$ search radius. We did not find any reliable $B$-band size for CGCG 247-020, IRASF $08339+6517$, and IRASF $12132+5313$, nor did we find an NIR size for NGC 5256, NGC 5331, and MCG +07-23-019. All these galaxies belong to the LIRGs subsample. No correction was applied to the luminosities and diameters for internal extinction or inclination of the host galaxies. Following RBGS we adopt $\Omega_{M}=$ $0.3, \Omega_{\Lambda}=0.7$, and $H_{0}=75 \mathrm{~km} \mathrm{~s}^{-1} \mathrm{Mpc}^{-1}$. These values are slightly different than recent estimates from the Wilkinson Microwave Anisotropy Probe (WMAP; Spergel et al. 2003). Table 1 includes a partial list of low-luminosity SFGs showing only cool and warm sources. In the table galaxies are sorted in ascending order of FIR color (i.e., galaxy 1 has the lowest FIR color) for all luminosity classes (see the electronic edition of the Journal for the complete list of galaxies).

\section{UNCERTAINTY IN PARAMETERS}

We use $L_{\mathrm{TIR}}$ as a photometric measure instead of $L_{\mathrm{FIR}}$, since the former is based on all four fluxes measured by IRAS and thus takes contributions from almost the entire IR range (Sanders \& Mirabel 1996). The median uncertainties associated with the IRAS flux densities are $\sim 5 \%$ and $\sim 3 \%$ at 12 and $25 \mu \mathrm{m}$, respectively, and $\sim 1 \%$ in both far-infrared bands. This leads to a $2 \%$ uncertainty in the FIR color of a galaxy, which is much smaller than the spread in mean color in a given luminosity. The total IR flux of a galaxy is a weighted sum of four IRAS flux densities. With the corresponding weighting factors given in Sanders \& Mirabel (1996), the median uncertainty in the total IR flux of a galaxy is $\sim 1 \%$.

Distances for the galaxies are taken from the RBGS. Most of the RBGS distance estimates come from redshift measurements, application of the Hubble law, and correction for the Mould et al. (2000) flow model. However, some are primary (P) or secondary (S) distance estimates, as flagged in RBGS Table 1, that do not come from the Hubble flow and cosmic attractor model. Our sample contains 48 galaxies of such distance estimates, all of which belong to the SFGs subsample. A significant uncertainty to a galaxy 

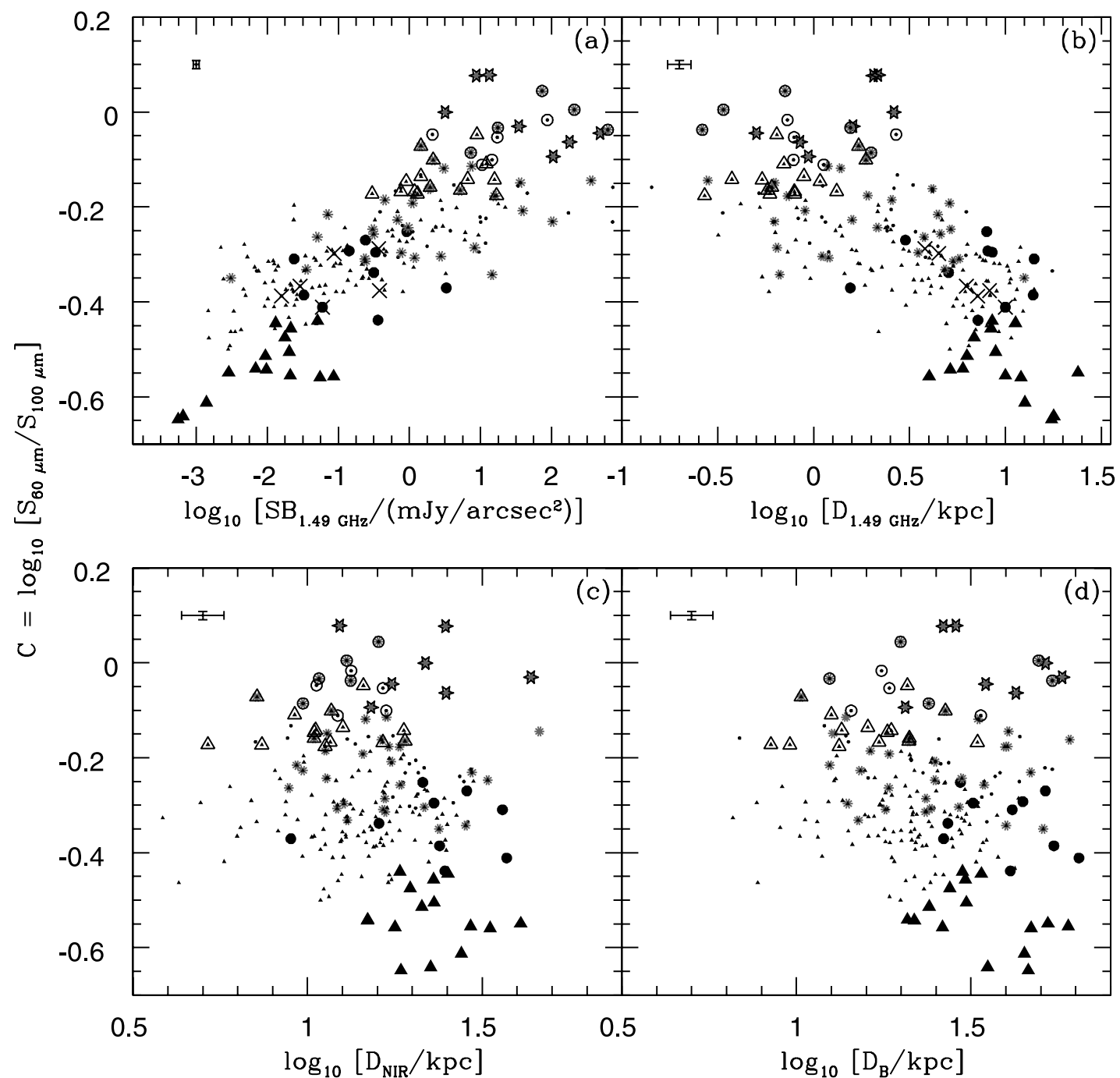

FIG. 2.-FIR color as a function of $(a)$ radio surface brightness, $(b)$ radio size, $(c)$ NIR size, and $(d)$ optical size. In each luminosity class cool sources have smaller surface brightness than the warmer ones ( $\operatorname{panel} a$ ). The FIR/radio emission in the cool systems is extended over a larger area compared to the warmer ones, where the emission is more compact (panel $b$ ). A weak trend between cool and warm sources can also be seen in the NIR and visual bands (panels $c$ and $d$ ). Large crosses (in panels $a$ and $b$ ) and asterisks represent, respectively, local submillimeter and interacting/disturbed galaxies. The symbols used are the same as in Fig. 1. A representative error bar is shown at the top left in each panel.

size and $L_{\mathrm{TIR}}$ would result from an uncertainty in the distance estimate. Surface brightness, being the distance-independent measure, would not be affected. The observed heliocentric radial velocities of sample galaxies are measured with $\sim 1 \%-2 \%$ accuracy (obtained from NED). The uncertainties associated with the model parameters, such as motions in the Local Group, Virgocentric infall, and Great Attractor infall, are high $(\geq 10 \%$; Mould et al. 2000). The uncertainty associated with other distance estimates is of similar magnitude. A galaxy optical angular size has less than $10 \%$ uncertainty, depending on the flattening of the disk, as given in the Third Reference Catalog (RC3; de Vaucouleurs et al. 1991). We assume a similar uncertainty in the NIR and radio bands (we note that it varies in different bands). Combining all of the preceding, we assign $\sim 15 \%$ and $\sim 20 \%$ uncertainty, respectively, to the estimate of physical size and $L_{\text {TIR }}$ of a galaxy.

The uncertainty in $1.49 \mathrm{GHz}$ surface brightness is due to the measurement errors in $1.49 \mathrm{GHz}$ flux density and angular size. According to CAH91, the contributions from rms confusion error and calibration error to the $1.49 \mathrm{GHz}$ flux density are smaller than the noise error. The rms radio map noise $\sigma_{n}$ is between 0.1 and $0.2 \mathrm{mJy}$ per beam solid angle $\Omega_{b} \sim \theta_{\text {beam }}^{2}$ (Condon 1987; CAH91).
The noise contribution to the flux density is $\sigma_{S} \approx \sigma_{n}\left(\Omega / \Omega_{b}\right)^{1 / 2}$ in $\mathrm{mJy}$, where $\Omega \sim \theta_{M}^{R} \times \theta_{m}^{R}$ is the solid angle covered by the source and $\theta_{M}^{R}, \theta_{m}^{R}$ are the deconvolved major $(M)$ and minor $(m)$ axes of a radio image. Radio maps in CAH91 range in FWHM angular resolution from 1.5 to $60^{\prime \prime}$ depending on the source's apparent size. Using $\sigma_{n}=0.2 \mathrm{mJy}$ for each galaxy gives an uncertainty of $\sim 2 \%$ in $1.49 \mathrm{GHz}$ flux density. Uncertainty in radio size varies with beam resolutions, but it is within $5 \%$ in all cases. Taking this upper limit we find $\sim 10 \%$ uncertainty in the surface brightness coming from $1.49 \mathrm{GHz}$ flux density and angular size.

\section{RESULTS}

Figure 1 shows the FIR color-IR luminosity diagram for an IRASS $S_{60 \mu \mathrm{m}}>1.2$ Jy sample of $\sim 4700$ sources (Fisher et al. 1995) (Fig. 1a) and our sample of 213 galaxies with $S_{60 \mu \mathrm{m}}>5.4 \mathrm{Jy}$ (Fig.1b). The former sample is shown after removing 330 spurious cold luminous galaxies as pointed out by Chapman et al. (2003). In both panels the horizontal bars and small crosses represent the median and mean color with a $1 \sigma$ error bar. These panels highlight the fact that galaxy FIR color and total IR luminosity follow a broad correlation with a large spread in different flux-limited 
TABLE 2

Correlation Statistics from Pearson's Correlation Test

\begin{tabular}{|c|c|c|c|c|c|c|c|c|}
\hline \multirow[b]{2}{*}{ Galaxy Class } & \multicolumn{2}{|c|}{$C-\mathrm{SB}_{R}$} & \multicolumn{2}{|c|}{$C-D_{R}$} & \multicolumn{2}{|c|}{$C-D_{\mathrm{NIR}}$} & \multicolumn{2}{|c|}{$C-D_{B}$} \\
\hline & $r$ & $P$ & $r$ & $P$ & $r$ & $P$ & $r$ & $P$ \\
\hline SFGs+LIRGs+ULIRGs......... & 0.77 & $1.0 \mathrm{E}-08$ & -0.65 & $2.14 \mathrm{E}-07$ & -0.14 & $4.74 \mathrm{E}-02$ & -0.11 & $1.15 \mathrm{E}-01$ \\
\hline 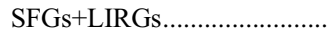 & 0.77 & $1.0 \mathrm{E}-08$ & -0.67 & $1.83 \mathrm{E}-08$ & -0.22 & $1.91 \mathrm{E}-03$ & -0.19 & $5.33 \mathrm{E}-03$ \\
\hline
\end{tabular}

Notes.-Correlation statistics for FIR color $C$ vs. all other parameters in Fig. 2. Here $r$ is the correlation coefficient and $P$ is the probability that correlation could arise from an uncorrelated sample. The confidence is $1-P$. The galaxy $1.49 \mathrm{GHz}$ surface brightness and size are represented by $\mathrm{SB}_{R}$ and $D_{R}$. For both the $K_{s}$ and $B$ bands, the test is performed after removing galaxies with unknown sizes.

samples. In this figure small triangles and dots represent SFGs and LIRGs, respectively (Fig. 1b). For these two classes of galaxies solid symbols represent cool sources (around or below $1 \sigma$ from the mean $C$ ) and open symbols represent the warm sources (around or above $1 \sigma$ from the mean $C$ ). Open stars are used for the ULIRGs. Because of their limited number we are unable to separate these ultraluminous galaxies into cool and warm categories. In this panel large crosses represent local submillimeter galaxies (see below for a discussion of these galaxies).

Figure 2 shows color as a function of $\mathrm{SB}_{1.49 \mathrm{GHz}}$, as well as galaxy size in the radio, NIR, and optical bands. The choice of these parameters is based on conventional practice that suggests that radio size is a stand-in for FIR emission size, NIR size represents emission from old stellar population, and optical size accounts for the emission from a mixture of both young and old stars but complicated by dust. We have used the major axis to estimate the area of each object to reduce the effects of highly uncertain inclinations. The radio surface brightness is calculated as $S_{1.49 \mathrm{GHz}} /\left(\theta_{M} / 2\right)^{2}$.

Cool and warm galaxies separate clearly in the surface brightness versus color diagram (Fig. 2a). For each luminosity class, warm colors correlate with high surface brightness. Figure $2 b$ indicates that galaxies that are dominated by cool dust are large, massive spirals that are not strongly interacting or merging and presumably the ones with the least active star formation. Dust in these cool objects is also, on average, less centrally concentrated than in the more typical LIRGs and ULIRGs that are dominated by warm dust. There is a clear sequence in the progression of color with radio size: color increases systematically from normal/isolated disks toward merger/disturbed disks. The latter type of disks are shown by asterisk symbols. Figures $2 a$ and $2 b$ show that color is strongly correlated with both surface brightness and galaxy size at $1.49 \mathrm{GHz}$ (see Table 2 for correlation statistics). Note that the uncertainty in color or brightness is statistical in nature and does not include calibration error. However, it will not affect our results since incorporating calibration error will simply shift these physical parameters systematically.

The statistical trend in Figures $2 b$ and $2 c$ suggests that luminous cool galaxies, where older stars have greater spatial distribution, show a tendency to have cool dust distributed over larger volume. This may be interpreted as an indirect but interesting support of the idea that FIR light from cool galaxies is reprocessed emission of photons coming predominantly from the old population of stars. This is in accord with the expectation that dust heated by the nonionizing photons from a cooler population of stars might follow the smoothed distribution of older stars in the galaxy. In spite of a large scatter, we can see a moderate trend wherein warm sources have relatively smaller regions of NIR emission than their cool counterparts. Most of the ULIRGs (4/7) have larger NIR and $B$-band diameters than found among warm $(C>-0.2)$ LIRGs (Figs. $2 c$ and $2 d$ ). It is interesting that ULIRGs, which all have very warm dust $(C>-0.1)$, have NIR and $B$ diameters compa- rable to the subset of LIRGs with relatively cool dust temperatures $(C<-0.22)$. The data indicate that the ULIRGs have relatively large disks (e.g., they involve mergers of massive galaxies) with compact cores that dominate the FIR emission. It is also clear from Figure $2 c$ that warm LIRGs (large open circles) have systematically smaller NIR diameters than cool LIRGs (large filled circles). We conclude that some galaxies with relatively cool dust temperatures are LIRGs (rather than lower luminosity SFGs) because, despite having a lower average star formation rate (SFR) per unit area, they have on average larger total surface areas than warm LIRGs.

The optical size, representing the spatial distribution of stellar emission, shows no trend with color when the whole sample is considered (Fig. $2 d$ ). However, it improves for SFGs+LIRGs after removing ULIRGs from the sample (see Table 2 for correlation statistics). The scatter is large compared to Figures $2 b$ and $2 c$. All ULIRGs and a substantial number of warm SFGs and LIRGs are almost similar in optical size compared to their cool counterparts. All of these warm sources show signs of interaction such as a tidal tail or disturbed outer region, or the presence of neighbors.

With the exception of color and $\mathrm{SB}_{1.49} \mathrm{GHz}$, all of the physical parameters depend on the distance. Figure 2 demonstrates that color is tightly correlated with distance-independent measures. On the other hand, correlation between color and galaxy size is weak. There are at least three factors that can contribute to the broader distribution in color-size correlations (see Table 2 for correlation statistics). First, there may be large error in the distance measurement in spite of correction for non-Hubble flows since correlation between color and NIR or $B$-band surface brightness is relatively stronger compared to the respective sizes. Second, the optical diameter $D_{B}$ of interacting/merging galaxies will overestimate the actual size because of the inherent difficulty associated with size estimates of these sources. Removing the ULIRGs from the sample makes the color-size trend stronger. Third, varying scatters in color versus size plots reflect methodological differences in estimating angular size in different wavelengths. The scatter would have been reduced if galaxy sizes were estimated in all wavelengths in a systematic manner, e.g., at the same brightness level.

Figure 3 shows color as a function of galaxy (optical) morphology. We use the RC3 classification and divide galaxies into three broad categories: unbarred (A), transitional (AB), and barred (B). In each morphology bin, we take $\mathrm{S} 0$ ?-S0a-Sa-Sb-Sbc sources as early type, and S?-Sc-Scd-Sd-I-Pec sources as late type. The earlyand late-type division is made to be consistent with earlier studies that have found fundamental differences in the properties of early- and late-type galaxies, especially barred galaxies (Combes \& Elmegreen 1993; Ho et al. 1997; Sakamato et al. 1999; Sheth et al. 2005).

We did not find any classification for 11 LIRGs, and classification is also uncertain for the seven ULIRGs (see Table 1 in 


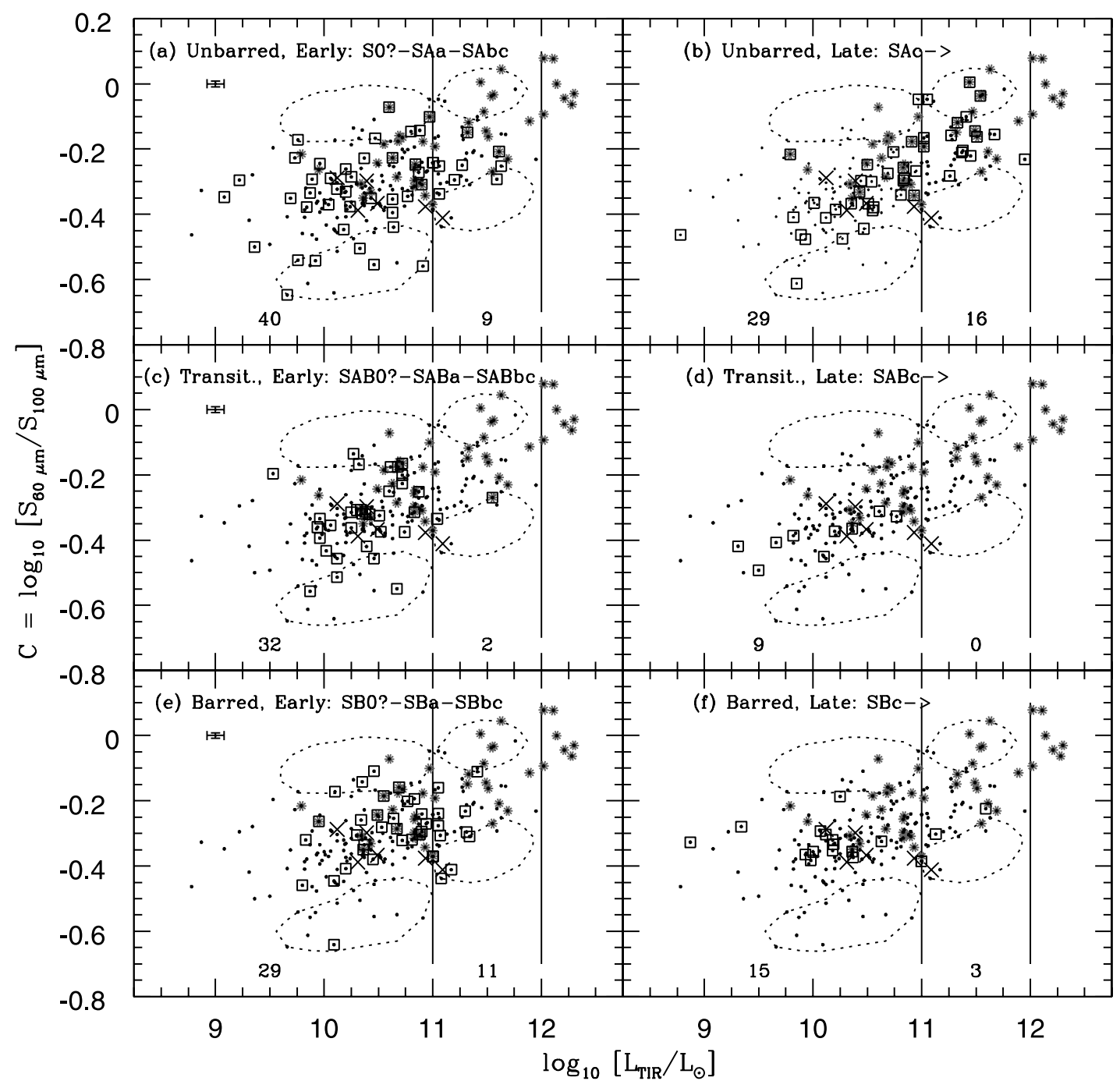

FIG. 3.-FIR color as a function of galaxy (optical) morphology. Dotted closed curves show the regions of cool and warm sources. Large crosses and asterisks represent, respectively, local submillimeter and interacting/disturbed galaxies. Representative error bar is shown at the top left and the number of galaxies with different morphologies are shown at the bottom of each panel. Only those galaxies, 154 SFGs and 41 LIRGs, that have morphological classification available in the RC 3 catalog are shown. Note from the figure that both transitional and barred spirals appear to be low-luminosity SFGs, whereas high-luminosity warm galaxies are predominantly unbarred (i.e., normal spirals). Cool and warm sources, in a given luminosity class, contain spirals with various morphologies.

the electronic edition of the Journal for these galaxies). As a result, Figure 3 shows 154 SFGs and 41 LIRGs divided into SA-, SAB-, and SB-type sources. This figure illustrates that high-luminosity LIRGs are mostly unbarred spirals (Figs. $3 a$ and $3 b$ ). The transitional and barred spirals, in general, fall into the class of lowluminosity SFGs (Figs. $3 c-3 d$ and $3 e-3 f$ ). The essence of this figure is the following: for SFGs, late-type spirals stay close to the mean color (Figs. $3 d$ and $3 f$ ). Early-type spirals, on the other hand, are responsible for the scatter in the color-luminosity diagram (Figs. $3 a, 3 c$, and $3 e$ ). Interestingly, cool sources consist of early-type unbarred and transitional galaxies, whereas warm sources consist of early types of all three categories. In other words, low-luminosity barred galaxies, in general, show a tendency to have higher color temperature. For LIRGs, late-type unbarred galaxies are predominately warm (Fig. $3 b$ ). This is not unexpected since late-type galaxies are highly irregular systems with higher SFRs, which results in warmer colors. Early-type LIRGs are systematically cooler (Figs. $3 a, 3 c$, and $3 e$ ). Both transitional and barred spirals are extremely rare in this luminosity class.

In a recent study, Sheth et al. (2005) showed that late-type barred spirals are less centrally concentrated than early types and that a significant subset of early-type barred spirals have little or no gas within the bar region. This observation has been explained as the result of higher mass accretion rates in the past in early-type barred spirals, wherein the large amount of gas driven inward by the bar has already been converted into stars. They suggested that these galaxies are in the poststarburst phase. The IR luminosity of the Sheth et al. sample of 44 galaxies spans the range $10^{9}<L_{\mathrm{TIR}} / L_{\odot}<10^{11}$. Thus the galaxies of the Sheth et al. sample fall in the class of star-forming galaxies (SFGs) according to the luminosity range used in our study. The observation that early-type barred spirals are in the poststarburst phase gives a plausible explanation for these galaxies being low-luminosity star-forming systems.

Note that the RC3 classifications used here are based on optical images that are strongly affected by dust obscuration in our FIRselected galaxy sample. More recent studies at NIR wavelengths indicate the presence of stellar bars in a large fraction of disk galaxies that appear unbarred at optical wavelengths (Eskridge et al. 2000). In addition, when one extends the bar detection sensitivity to a low relative amplitude of $\sim 3 \%$ in the $K$ band, nearly $90 \%$ of a sample of optically unbarred (SA) spirals contain stellar 
bars (Grosbol et al. 2004). Therefore, the fractions of transition $(\mathrm{SAB})$ and barred (SB) galaxies is likely to be much higher than is indicated by the RC3 classifications, and the optical bar fractions may be telling us more about the relative extinction by dust at optical wavelengths than the intrinsic stellar distributions. An improved understanding of this issue requires detailed analysis of near-IR images for a large sample of LIRGs and ULIRGs.

\section{DISCUSSION}

It is necessary to address whether the large scatter in the FIR color-luminosity diagrams is real (intrinsic to the physics of the objects) or an artifact of the observations. Figure 1 shows the data from the IRAS sample of Fisher et al. $\left(S_{60 \mu \mathrm{m}}>1.2 \mathrm{Jy}\right.$; Fig. $\left.1 a\right)$ and data from the RBGS $\left(S_{60 \mu \mathrm{m}}>1.2 \mathrm{Jy} ;\right.$ Fig. $\left.1 b\right)$. The main objective of the RBGS was to produce more accurate fluxes for the many large, nearby galaxies resolved by IRAS, by recovering extended emission that was not represented in the (underestimated) flux densities reported for such objects in the IRAS Point Source Catalog (PSC) and Faint Source Catalog (FSC). The RBGS also added some objects missed in the previous BGS compilations. This problem is minimal for the fainter objects in the $1.2 \mathrm{Jy}$ sample, which on average are more distant and therefore smaller than the RBGS objects, with little or no flux missed in the published $\mathrm{PSC} / \mathrm{FSC}$ flux densities. The main conclusion we can draw from comparing Figure $1 a$ with Figure $1 b$ is that the scatter in the 5.24 Jy RBGS sample is consistent with the scatter in the Fisher et al. 1.2 Jy sample. The presence of more true statistical outliers ( $\geq 3 \sigma$ in each luminosity bin) in the 1.2 Jy sample compared to the $5.24 \mathrm{Jy}$ sample is expected from basic sampling statistics, as is the extension to higher $L_{\mathrm{TIR}}$ values due to sampling of a much larger volume of space in the $1.2 \mathrm{Jy}$ sample. As stated in previous sections, the uncertainty in the $L_{\mathrm{TIR}}$ values is dominated by the distance determinations $(\sim 15 \%)$, and the uncertainty in $C$ is relatively small (see the representative error bar in Fig. $1 a$ ). Therefore, we conclude that the dispersion in the $C$ - $L_{\mathrm{TIR}}$ diagram is intrinsic and related to the diversity of physical conditions of the ISM in the galaxies.

To understand the physical origin of the color-luminosity diagram it is necessary to have detailed knowledge of these cool and warm galaxies. Questions can be raised such as, In a given luminosity range, what are the possible (external or internal) mechanisms that would cause some galaxies to have cool color temperature compared to the majority of the galaxies? What are the basic connections between the photometric and structural properties of galaxies in this diagram? We proceed in light of the above queries and find that dust in the cool objects is less centrally concentrated than in the more typical LIRGs and ULIRGs that are dominated by more centrally concentrated warm dust. We also find that the optical disks of IR cool galaxies show a tendency to be more extended than those of the warmer ones. The color-size trend is relatively stronger at longer wavelengths. The extended emission in cool sources may well indicate heating by old stars, but it could also indicate simply wide-spread small star-forming regions scattered in a large dusty disk. Distinguishing between these aspects is beyond the scope of this study.

The color-luminosity trend shown in Figure 1 is observed in the local universe $(z<0.082)$. It has been demonstrated that the trend does not change out to $z \sim 1$ (Chapman et al. 2003), meaning that the dispersion of color in each luminosity bin does not vary significantly with redshift. However, given the presence of higher luminosity objects such as hyperluminous galaxies with $L_{\mathrm{TIR}} / L_{\odot}>$ $10^{13}$ in high-redshift samples that cover much larger volumes of space and earlier look-back times than local samples, from the present data we can only make such inferences for objects with
$L_{\mathrm{TIR}} / L_{\odot} \leq 10^{12.5}$. In this respect, this correlation, therefore, reveals something quite profound: in the absence of any structural information on high- $z$ luminous galaxies, we can get a rough idea about the extent of the disks if we simply know the FIR colors and luminosities of these galaxies. In spite of a large scatter, the trend suggests that cool galaxies are generally highly luminous because of extended disks. The warm galaxies may or may not have extended disks, depending on galaxy environment and/or internal processes.

Current submillimeter/millimeter surveys have discovered a new population of $z>1$ submillimeter galaxies, contributing up to $50 \%$ of the extragalactic background light (Smail et al. 1997; Barger et al. 1998; Hughes et al. 1998; Borys et al. 2003). There are two possible explanations of these galaxies: they are either protospheroids with high SFR $\geq 10^{3} M_{\odot} \mathrm{yr}^{-1}$ (Dunlop 2001) or disks dominated in the submillimeter by infrared cirrus heated by their interstellar radiation field, rather than intense star formation (Rowan-Robinson 2001; Efsthathiou \& Rowan-Robinson 2003, hereafter ER03; King \& Rowan-Robinson 2003). ER03 have modeled cirrus emission for a selection of local $(z<0.02)$ and high- $z(>1)$ submillimeter galaxies and found excellent agreement in all cases. Their work suggests that the submillimeter count and background can be understood in terms of cirruslike emission (with effective dust temperature $<30 \mathrm{~K}$ ), rather than invoking dusty ultraluminous starburst galaxies, e.g., Arp 220 in our local universe, to be the representative of distant submillimeter galaxies. The significance of ER03's work is that if the cool component dominates the observed submillimeter fluxes of these high- $z$ submillimeter/millimeter wave survey sources, the emission should be extended rather then compact (i.e., centrally condensed). We attempt to explore this aspect of submillimeter galaxies in the context of our study by analyzing the $1.49 \mathrm{GHz}$ emission of the local sample of ER03 since only these galaxies are available in the RBGS.

The local sample of ER03 includes UGC 0903, NGC 0958, NGC 1667, NGC 2990, UGC 5376, NGC 5962, and NGC 6181. We remove NGC 2990 from the ER03 sample because it has $S_{60 \mu \mathrm{m}}<5.24 \mathrm{Jy}$, placing it below the flux limit of the RBGS. Except for NGC 1667 and UGC 5376, the other four galaxies are present in our sample. The IR and radio information for these two galaxies is obtained respectively from the RBGS and the NRAO VLA Sky Survey catalog (NVSS; Condon et al. 1998). The FIR color and total IR luminosities of these six galaxies (shown by large crosses) are similar to normal SFGs and cool LIRGs in our sample (Fig. 1b). We find that these galaxies indeed have large radio (FIR) disks. The $1.49 \mathrm{GHz}$ radio source diameters of these galaxies range from $\sim 4$ to $20 \mathrm{kpc}$, which is comparable to cool LIRGs and even normal SFGs (those not defined in this study as unusually cool or warm). This is in stark contrast to the warm SFGs and LIRGs that have radio sizes less than $\sim 3$ kpc (see Fig. $2 b$ ).

The FIR cool high- $z$ submillimeter galaxies of the ER03 sample show a few $\times 10^{13} L_{\odot}$, several hundred times more luminous than these local examples. Assuming that these galaxies are scaled-up versions of local cool examples, where disk size increases at constant surface brightness to account for the increased luminosity, we find that their radio (and FIR) disks must range up to $100 \mathrm{kpc}$ or more in size. While rare, local galaxies with similar sizes do exist, as in the case of low surface brightness, $\mathrm{H}$ I-rich galaxies (Sprayberry et al. 1995; Bothun et al. 1997; Matthews et al. 2001). At earlier epochs the cold submillimeter galaxies may be single large disks able to regulate their star formation at the modest level required for these luminosities and FIR colors. But what do we know from observations about the extent of 
FIR/radio emission in these distant submillimeter sources, especially the FIR cool ones?

For the assumed cosmology in this study, the linear disk size of $100 \mathrm{kpc}$ corresponds to an angular size of $>7^{\prime \prime}$ for $z>1 \mathrm{sub}$ millimeter galaxies. On the other hand, the reported size of submillimeter galaxies in the redshift range $1 \lesssim z \lesssim 4$ is significantly smaller, 1".5 (Ivison et al. 2002; Iono et al. 2006; Tacconi et al. 2006). This suggests that the objects measured are best understood as a scaled-up version of local FIR warm ULIRGs rather than cool extended disks (Tacconi et al. 2006), since nothing is known about their FIR colors. While cold ULIRGs had been detected in the redshift range $0.4<z<1$, very little is known about their morphology or geometry (Chapman et al. 2002). However, the recently reported optical properties of BzK 15504 (Genzel et al. 2006) make it a very likely cool luminous galaxy. At $z=2.38$, its disk extends out to $\sim 10 \mathrm{kpc}$ and has elevated distributed star formation throughout, in addition to an accreting central black hole. The estimated total star formation rate for this galaxy is $140_{-80}^{+100} M_{\odot} \mathrm{yr}^{-1}$ (Genzel et al. 2006), suggesting that it is a ULIRG with $L_{\mathrm{TIR}} \gtrsim 10^{12} L_{\odot}$.

The relative frequency of occurence of FIR cool objects as a function of redshift is still unknown, and the detection efficiency will decrease as the source extent increases. Tacconi et al. have measured eight objects, all of which were compact. Their results only place a limit on the effective dust temperature $(39 \pm 3 \mathrm{~K}$ for $\beta=1.5$ ) of submillimeter galaxies rather than the frequency of cool objects. As precise redshifts and more accurate rest-frame FIR fluxes of high- $z$ galaxies become available, placing these submillimeter/millimeter galaxies in the context of the FIR color-luminosity diagram will help shed light on their structure. Combining that with kinematic information may constrain further the nature and evolving path of these galaxies.

Warm LIRGs and ULIRGs are usually associated with intense bursts of star formation. These galaxies are known to have higher SFRs and star formation efficiencies (SFEs) than lower luminosity galaxies. These parameters are defined in such a manner that the SFR is directly related to the $L_{\mathrm{TIR}}$ (Kennicutt 1998) whereas the SFE is the $L_{\mathrm{TIR}}$ normalized by the molecular hydrogen mass (Young 1999). It has been noted that along the Hubble sequence, the SFR increases toward late types, which could be due to dynamical instability increasing with decreasing bulge-to-disk ratio (Combes 2001). This trend is generally observed among the optically unbarred spirals in the present study as well, whereas galaxies with warmer FIR colors and hence higher SFRs are less likely to be transitional (SAB) or barred (SB) galaxies (see Fig. 2).

Questions can also be raised such as, What types of morphological features do the cool and warm galaxies have and how could these features be linked with the FIR color-luminosity correlation? We find that the LIRGs in our sample are mostly late-type unbarred spirals. The transitional and barred spirals, in general, fall into the class of low-luminosity SFGs. Among LIRGs, the late-type unbarred spirals are predominately warm, and the early-type unbarred and barred are systematically cooler. One probable reason for the transitional and barred spirals being low-luminosity star- forming galaxies is that they are in the poststarburst phase, where the large amount of gas driven inward by the bar have already been converted into stars, consistent with the findings of Sheth et al. (2005).

Finally, we note that the low-luminosity early-type unbarred (SA) and transitional (SAB) spirals are responsible for the large scatter in the $C-L_{\mathrm{TIR}}$ diagram. It may be that in spite of our selection process, we have dust-enshrouded AGNs in the sample. These sources could span the entire luminosity range considered and could contribute to the scatter in this diagram. On the other hand, this scatter may also arise from more variability in the SFR and star formation behavior, e.g., short episodic starbursts or more possible configurations, e.g., the large bulges and small star formation disks of early-type sources.

\section{CONCLUSIONS}

We have analyzed a sample of 213 nearby IR galaxies to study the correlations of galaxy FIR color with several photometric and physical parameters. The set of measures include total IR luminosity, radio surface brightness, and radio, NIR, and optical sizes. Our objective is to understand cool and warm IR sources using various correlations. We find that galaxy radio surface brightness is well correlated with FIR color, whereas size is less well correlated with color. A weak color-size correlation signals a significant uncertainty associated with the distance measurements of sample galaxies. It may also reflect methodological differences in estimating angular size in different wavelengths. We also find that late-type galaxies (from Sc and beyond) of all morphology classes show less scattering than early types in the color-luminosity diagram. Our study shows that dust in the cool IR sources is probably less centrally concentrated than in the more typical luminous and ultraluminous IR galaxies that are dominated by more centrally concentrated warm dust. We believe this result has significant implication in terms of the FIR color-luminosity diagram: in the absence of any structural information on high- $z$ luminous sources, one can get a rough estimate of the extent of the disks if one simply knows the FIR colors and luminosities of galaxies.

We thank the referee Jim Houck for insightful comments, and Thomas Jarrett for many useful communications. N. R. thanks Ranga Ram Chary, Roc Cutri, Justin Howell, Guilain Lagache, Seppo Laine, Naveen Reddy, Kevin Xu, Min Su Yun, and Zang Wang for discussions. N. R. gratefully acknowledges the support of a research associateship administered by the National Research Council (up to 2005 December) and the Oak Ridge Associated Universities (currently) during this research. This study has made use of the NASA/IPAC Extragalactic Database (NED), which is operated by the Jet Propulsion Laboratory, California Institute of Technology, under contract with the National Aeronautics and Space Administration, and the LEDA database in France.
Alton, P. B., et al. 1998, A\&A, 335, 807

Andreani, P., \& Franceschini, A. 1992, A\&A, 260, 89 1996, MNRAS, 283, 85

Barger, A. J., Cowie, L. L., Sanders, D. B., Fulton, E., Taniguchi, Y., Sato, Y.,

Kawara, K., \& Okuda, H. 1998, Nature, 394, 248

Barvainis, R., Antonucci, R., \& Coleman, P. 1992, ApJ, 399, L19

Beck, R., \& Golla, G. 1988, A\&A, 191, L9

Bicay, M. D., \& Helou, G. 1990, ApJ, 362, 59

Bicay, M. D., Helou, G., \& Condon, J. J. 1989, ApJ, 338, L53

\section{REFERENCES}

Borys, C., Chapman, S. C., Halpern, M., \& Scott, D. 2003, MNRAS, 344, 385

Bothun, G. D., Impey, C. D., \& McGaugh, S. S. 1997, PASP, 109, 745

Bothun, G. D., Lonsdale, C. J., \& Rice, W. 1989, ApJ, 341, 129

Carico, D. P., Sanders, D. B., Soifer, B. T., Matthews, K., \& Neugebauer, G. 1990, AJ, 100, 70

Chapman, S. C., Helou, G., Lewis, G. F., \& Dale, D. A. 2003, ApJ, 588, 186 Chapman, S. C., Smail, I., Ivison, R. J., Helou, G., Dale, D. A., \& Lagache, G. 2002, ApJ, 573, 66 
Combes, F. 2001, in ASP Conf. Ser. 249, The Central Kiloparsec of Starbursts and AGN, ed. J. H. Knapen, J. E. Beckman, I. Sholsman, \& T. J. Mahoney (San Francisco: ASP), 475

Combes, F., \& Elmegreen, B. G. 1993, A\&A, 271, 391

Condon, J. J. 1987, ApJS, 65, 485

Condon, J. J., Anderson, M. L., \& Helou, G. 1991, ApJ, 376, 95 (CAH91)

Condon, J. J., Cotton, W. D., Greisen, E. W., Yin, Q. F., Perley, R. A., Taylor, G. B., \& Broderick, J. J. 1998, AJ, 115, 1693

Dale, D., Helou, G., Contursi, A., Silbermann, N. A., \& Kolhatkar, S. 2001, ApJ, 549, 215

de Grijp, M. H. K., Miley, G. K., Lub, J., \& de Jong, T. 1985, Nature, 314, 240

de Vaucouleurs, G., de Vaucouleurs, A., Corwin, H. G., Buta, R. J., Paturel, G., \& Fouqué, P. 1991, Third Reference Catalog of Bright Galaxies (New York: Springer)

Domingue, D. L., Keel, W. C., Ryder, S. D., \& White, R. E., III. 1999, AJ, 118, 1542

Dunlop, J. 2001, in Deep Millimeter Surveys: Implications for Galaxy Formation and Evolution, ed. J. D. Lowenthal \& D. H. Hughes (Singapore: World Scientific), 11

Dunne, L., \& Eales, S. 2001, MNRAS, 327, 697

Dunne, L., Eales, S., Edmunds, M., Ivison, R., Alexander, P., \& Clements, D. L. 2000, MNRAS, 315, 115

Efstathiou, A., \& Rowan-Robinson, M. 2003, MNRAS, 343, 322 (ER03)

Eskridge, P. B., et al. 2000, AJ, 119, 536

Fisher, K. B., et al. 1995, ApJS, 100, 69

Genzel, R., et al. 1998, ApJ, 498, 579 2006, Nature, 442, 786

Grosbol, P., Patsis, P. A., \& Pompei, E. 2004, A\&A, 423, 849

Haas, M., Klass, U., Coulson, I., Thommes, E., \& Xu, C. 2000, A\&A, 356, L83

Harwit, M., Houck, J. R., Soifer, B. T., \& Palumbo, G. G. C. 1987, ApJ, 315, 28

Helou, G. 1986, ApJ, 311, L33

Helou, G., Soifer, B. T., \& Rowan-Robinson, M. 1985, ApJ, 298, L7

Ho, L. C., Filippenko, A. V., \& Sargent, W. L. W. 1997, ApJ, 487, 591

Hughes, D. H., et al. 1998, Nature, 394, 241

Iono, D., et al. 2006, ApJ, 640, L1

Ivison, R. J., et al. 2002, MNRAS, 337, 1

Jarrett, T. H. 2000, PASP, 112, 1008

Jarrett, T. H., Chester, T., Cutri, R., Schneider, S., Skrutskie, M., \& Huchra, J. P. 2000, AJ, 119, 2498

Kennicutt, R. C. 1998, ApJ, 498, 541

Kim, D. C., \& Sanders, D. B. 1998, ApJS, 119, 41

King, A. J., \& Rowan-Robinson, M. 2003, MNRAS, 339, 260
Lawrence, A., Walker, D., Rowan-Robinson, M., Leech, K. J., \& Penston, M. V. 1986, MNRAS, 219, 687

Lonsdale, C., Smith, H., \& Lonsdale, C. 1993, ApJ, 405, L9

Lonsdale Persson, C. J., \& Helou, G. 1987, ApJ, 314, 513

Lutz, D., Spoon, H. W. W., Rigopoulou, D., Moorwood, A. F. M., \& Genzel, R. 1998, ApJ, 505, L103

Marsh, K. A., \& Helou, G. 1995, ApJ, 445, 599

Matthews, L. D., van Driel, W., \& Monnier-Ragaigne, D. 2001, A\&A, 365, 1 Mould, J. R., et al. 2000, ApJ, 529, 786

Murphy, E. J., et al. 2006, ApJ, 638, 157

Rowan-Robinson, M. 2001, ApJ, 549, 745

Sakamato, K., Okumura, S. K., Ishizuki, S., \& Scoville, N. Z. 1999, ApJS, 124, 403

Sanders, D. B., Mazzarella, J. M., Kim, D.-C., Surace, J. A., \& Soifer, B. T. 2003, AJ, 126, 1607

Sanders, D. B., \& Mirabel, I. F.1996, ARA\&A, 34, 749

Sanders, D. B., Soifer, B. T., Elias, J. H., Madore, B. F., Matthews, K., Neugebauer, G., \& Scoville, N. Z. 1988, ApJ, 325, 74

Sheth, K., Vogel, S. N., Regan, M. W., Thornley, M. D., \& Teuben, P. 2005, ApJ, 632, 217

Siebenmorgen, R., Krügel, E., \& Chini, R. 1999, A\&A, 351, 495

Smail, I., Ivison, R. J., \& Blain, A. W. 1997, ApJ, 490, L5

Spergel, D. N., et al. 2003, ApJS, 148, 175

Soifer, B. T., Boehmer, L., Neugebauer, G., \& Sanders, D. B. 1989, AJ, 98, 766

Soifer, B. T., \& Neugebauer, G. 1991, AJ, 101, 354

Soifer, B. T., Sanders, D. B., Madore, B. F., Neugebauer, G., Danielson, G. E., Elias, J. H., Lonsdale, C. J., \& Rice, W. L. 1987, ApJ, 320, 238

Soifer, B. T., Sanders, D. B., Neugebauer, G., Danielson, G. E., Lonsdale, C. J., Madore, B. F., \& Persson, S. E. 1986, ApJ, 303, L41

Soifer, B. T., et al. 1984, ApJ, 278, L71

Sopp, H. M., \& Alexander, P. 1991, MNRAS, 251, 112 1992, MNRAS, 259, 425

Sprayberry, D., Impey, C. D., Bothun, G. D., \& Irwin, M. J. 1995, AJ, 109, 558 Stickel, M., et al. 2000, A\&A, 359, 865

Tacconi, L. J., et al. 2006, ApJ, 640, 228

Trewhella, M., Davis, J. I., Alton, P. B., Bianchi, S., \& Madore, B. 2000, ApJ, 543,153

Wang, Z., \& Helou, G. 1992, ApJ, 398, L33 (WH92)

Young, J. S. 1999, ApJ, 514, L87

Zink, E. C., Lester, D. F., Doppmann, G., \& Harvey, P. M. 2000, ApJS, 131, 413 